\title{
Human adenovirus binding to host cell receptors: a structural view
}

\author{
Aleksandra Cecylia Stasiak ${ }^{1}\left[\right.$ D $\cdot$ Thilo Stehle $^{1,2}$
}

Received: 16 August 2019 / Accepted: 14 November 2019 / Published online: 29 November 2019

(c) The Author(s) 2019

\begin{abstract}
Human Adenoviruses (HAdVs) are a family of clinically and therapeutically relevant viruses. A precise understanding of their host cell attachment and entry mechanisms can be applied in inhibitor design and the construction of targeted gene delivery vectors. In this article, structural data on adenovirus attachment and entry are reviewed. HAdVs engage two types of receptors: first, an attachment receptor that is bound by the fibre knob protein protruding from the icosahedral capsid, and next, an integrin entry receptor bound by the pentameric penton base at the capsid vertices. Adenoviruses use remarkably diverse attachment receptors, five of which have been studied structurally in the context of HAdV binding: Coxsackie and Adenovirus Receptor, CD46, the glycans GD1a and polysialic acid, and desmoglein-2. Together with the integrin entry receptors, they display both symmetrical and asymmetrical modes of binding to the virus as demonstrated by the structural analyses reviewed here. The diversity of HAdV receptors contributes to the broad tropism of these viruses, and structural studies are thus an important source of information on HAdV-host cell interactions. The imbalance in structural data between the more and less extensively studied receptors remains to be addressed by future research.
\end{abstract}

Keywords Human adenovirus $\cdot$ Structural biology $\cdot$ X-ray crystallography $\cdot$ Cryoelectron microscopy $\cdot$ Review

\section{Introduction}

\section{Human adenoviruses as pathogens and therapeutics}

Human Adenoviruses (HAdVs) are a family of non-enveloped double-stranded deoxyribonucleic acid (dsDNA) viruses with genomes of about 35 kilobases $(\mathrm{kb})$ [1]. They are causative agents of a wide range of illnesses, such as conjunctivitis, gastroenteritis and respiratory infections [2]. Over 100 types of HAdVs, classified into seven groups

Edited by Volkhard A. J. Kempf.

This article is published as part of the Special Issue on ViBrANTITN, funded by the European Union's Horizon 2020 Research and Innovation Programme under the Marie Skłodowska-Curie Grant Agreement No. 765042.

Thilo Stehle

thilo.stehle@uni-tuebingen.de

1 Interfaculty Institute of Biochemistry, University of Tübingen, Hoppe-Seyler-Str. 4, 72076 Tübingen, Germany

2 Department of Pediatrics, Vanderbilt University School of Medicine, Nashville, TN 37232, USA
(A-G), have been reported to the HAdV Working Group (http://hadvwg.gmu.edu/). Viruses among these groups vary in pathology and molecular characteristics, for instance receptor specificity and host cell tropism $[3,4]$.

Due to their large genome capacity and the ability to infect different cell types, HAdVs have been extensively studied as gene delivery vectors, and HAdV family members have been used in hundreds of clinical trials in oncology, gene therapy, and vaccinology $[5,6]$. A detailed understanding of HAdV host cell attachment and entry mechanisms facilitates the design of vectors with a particular tropism, as well as the rational design of antiviral compounds. Structural biology techniques, in particular, can provide information on the molecular details of virus binding to host cell, and can identify strategies for the disruption of such interactions. So far, structural analyses of HAdV particles and their components have revealed symmetrical and asymmetrical binding modes to HAdV receptors, and these will be reviewed below.

\section{HAdV attachment and entry}

The HAdV capsid possesses $T=25$ icosahedral symmetry, and consists of three major proteins: the hexon, the penton base and the fibre (see Fig. 1) [2,7]. Both the fibre and 


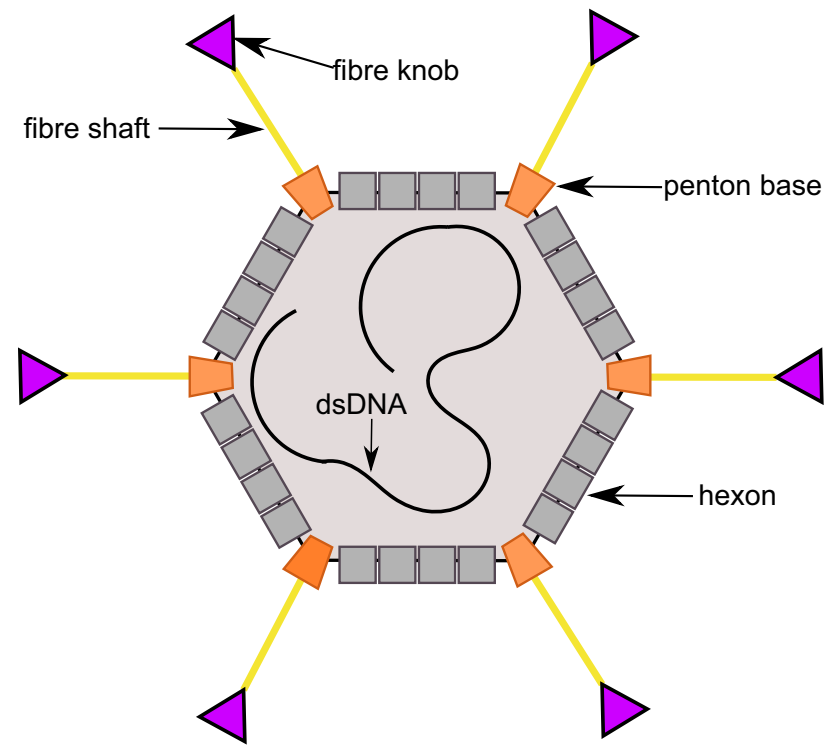

Fig. 1 A schematic drawing of Human Adenovirus, showing the major capsid proteins that interact with receptors. The fibre knob is in purple, the fibre shaft in yellow, the penton base in orange and the hexon in grey. The minor capsid proteins and non-structural proteins have been omitted for simplicity

penton base, forming the penton complex at the vertices of the icosahedron, can engage host cell receptors. The fibre can be divided into a C-terminal, globular structure, the "knob", which protrudes away from the capsid and mediates the initial interaction with attachment receptors, and an N-terminal, elongated "shaft" that anchors the fibre into the viral capsid. Binding of the knob to attachment receptors is followed by the penton base binding to the integrin entry receptor.

The fibre is a homotrimeric structure of between 60-80 kDa per monomer and its globular knob can engage different receptors [8-12]. The monomeric C-terminal fibre knob contains an eight-stranded antiparallel $\beta$-sandwich fold, comprised of two $\beta$-sheets, with multiple loops. The trimeric propeller-like knob is formed by intertwining $\beta$-sheets and has a deep depression at the centre, which thins into narrowing channels, exposing around $65 \%$ residues to the solvent $[8,13]$. The knob is mounted on the shaft, which consists of repeats of a hydrophobic 15-residue sequence assembled into a trimeric $\beta$-spiral [14]. The number of repeats, and hence the length of the shaft, varies between HAdV types. Residues at the very N-terminal region of the fibre knob form a tail that interacts non-covalently with the penton base [15].

Multiple HAdV attachment receptors have been identified: the cell surface proteins Coxsackie and Adenovirus Receptor (CAR) [16, 17], CD46 [10], and desmoglein-2 (DSG-2) [9], as well as the glycans GD1a [11] and polysialic acid [18]. The interactions of all five receptors with HAdV have been established using structural biology techniques and are the focus of this review. Other adenovirus receptors, such as heparan sulphate glycosaminoglycans [4], or factors IX [19] and X [20], have also been described, but we currently lack detailed structural information about their modes of binding to the virus.

Once HAdV has attached to the cell surface, the fibre starts disassociating from the capsid, exposing the penton base [21]. The penton base forms the vertex pentamer (see Fig. 1), which binds to the integrin entry receptor and exploits integrin-mediated signalling to enter the cell by endocytosis. HAdVs have been shown to use multiple types of integrins as their receptors, again highlighting their broad tropism [22].

The penton monomer consists of two domains: a jelly-roll domain proximal to the virion centre, and a distal insertion domain [23]. The latter contains the variable, highly mobile RGD loop, so named because it contains an arginine-glycine-aspartic acid tripeptide sequence motif. The integrinbinding RGD motif mediates binding between the integrin and the penton base, with the exception of group F HAdV-40 and -41 , where the interaction is presumed to take place in another manner due to the lack of this motif [24]. The length of the RGD loop varies significantly between strains, ranging from 36 amino acids for HAdV-12 to 99 amino acids for HAdV-5.

\section{HAdV Interactions with attachment receptors}

\section{Coxsackie and adenovirus receptor (CAR)}

CAR and CD46 have been the most extensively studied HAdV receptors, and their interactions with the fibre knob are particularly well understood. The first structure of a fibre knob bound to CAR was published in 1999, showing that the $\mathrm{N}$-terminal domain of CAR engages the fibre knob with an unusually discontinuous interface that features large, solvent-filled areas [8]. Structural information about fibre knob binding to CD46 became available in 2007, revealing a binding interface that spans the N-terminal two CD46 domains and that likely alters the conformation of unliganded CD46 [25]. The interactions of the fibre knobs with CAR and CD46 have been reviewed, for example, in [26]. Given the lack of extensive developments since then, this review will only give a brief summary.

CAR is a transmembrane protein mediating cell-cell adhesion [27]. It is a member of the Junctional Adhesion Molecule (JAM) family, and present in tight junctions and on epithelial cells at their lateral surfaces. CAR can form homodimers [28] or heterodimers with numerous extracellular and intracellular proteins, for instance fibronectin and 
JAM-L [29]. These interactions have been linked to processes such as $\mathrm{T}$ cell activation and cell adhesion, among others. CAR is a high-affinity receptor for HAdV groups A and $\mathrm{C}-\mathrm{F}$, as well as Coxsackievirus group B viruses [27].

CAR comprises two immunoglobulin-like extracellular domains, CAR-D1 and CAR-D2, both of which have been characterised structurally $[28,30]$. The D1 domain forms direct contacts with the fibre knob. The crystal structure of D1 in complex with the knob from HAdV-12 shows one D1 domain bound per knob monomer, with binding occurring at the interface of two monomers rather than at the central cavity (see Fig. 2) [8]. Interactions are mediated by four flexible loops of the knob, as was shown by both structural and mutational studies [31-33]. The AB loop of the fibre knob is responsible for over a half of the protein-protein contacts and is, therefore, an important determinant of CAR receptor specificity [8].

In a recent publication, the structures of the fibre knobs of HAdV-26 and 48 (group D) were solved, and computer modelling, surface plasmon resonance (SPR) and inhibition assays were used to examine binding to potential receptors, primarily CAR, CD46 and DSG-2 [34]. The structure of the fibre knob bound to CAR and CD46 was simulated in silico using homology models. A limited flexibility of loops involved in receptor binding in CAR and CD46 was postulated based on the X-ray data and interaction calculations. Binding of a lower affinity to CAR and no significant binding to CD46 and DSG-2 were proposed based on loop conformation and this was supported by competition inhibition assays.

\section{CD46 (human membrane cofactor protein)}

Human CD46, or membrane cofactor protein (MCP), is a ubiquitously expressed transmembrane glycoprotein involved in multiple processes, such as complement response inhibition, fertilisation, and adaptive immunity regulation through its extracellular domains $[35,36]$. CD46 is a member of the RCA (regulators of complement activation) family, and as such its role in binding complement proteins $\mathrm{C} 3 \mathrm{~b}$ and $\mathrm{C} 4 \mathrm{~b}$ is of particular importance since this interaction prevents the activation of complement response against autologous cells [36]. Complement system proteins are also pathogen receptors. CD46 can serve as a receptor for pathogenic bacteria and viruses, including Neisseria, measles, human herpesvirus-6, and some group B HAdVs [36]. RCA family members CD55 and CD21 serve as receptors for echoviruses [37] and Epstein-Barr virus [38], respectively.

The extracellular part of CD46 consists of four short consensus repeats (SCR, 1-4) and a serine, threonine and proline (STP)-rich region [39]. In an unbound form, SCR4 (proximal to cell membrane) is bent in relation to the almostlinearly arranged remaining subunits, forming a "hockey stick" shape. SCR1 and 2 have been identified as the domains interacting with the HAdV knob. When only these two subunits are expressed, a greater degree of flexibility is

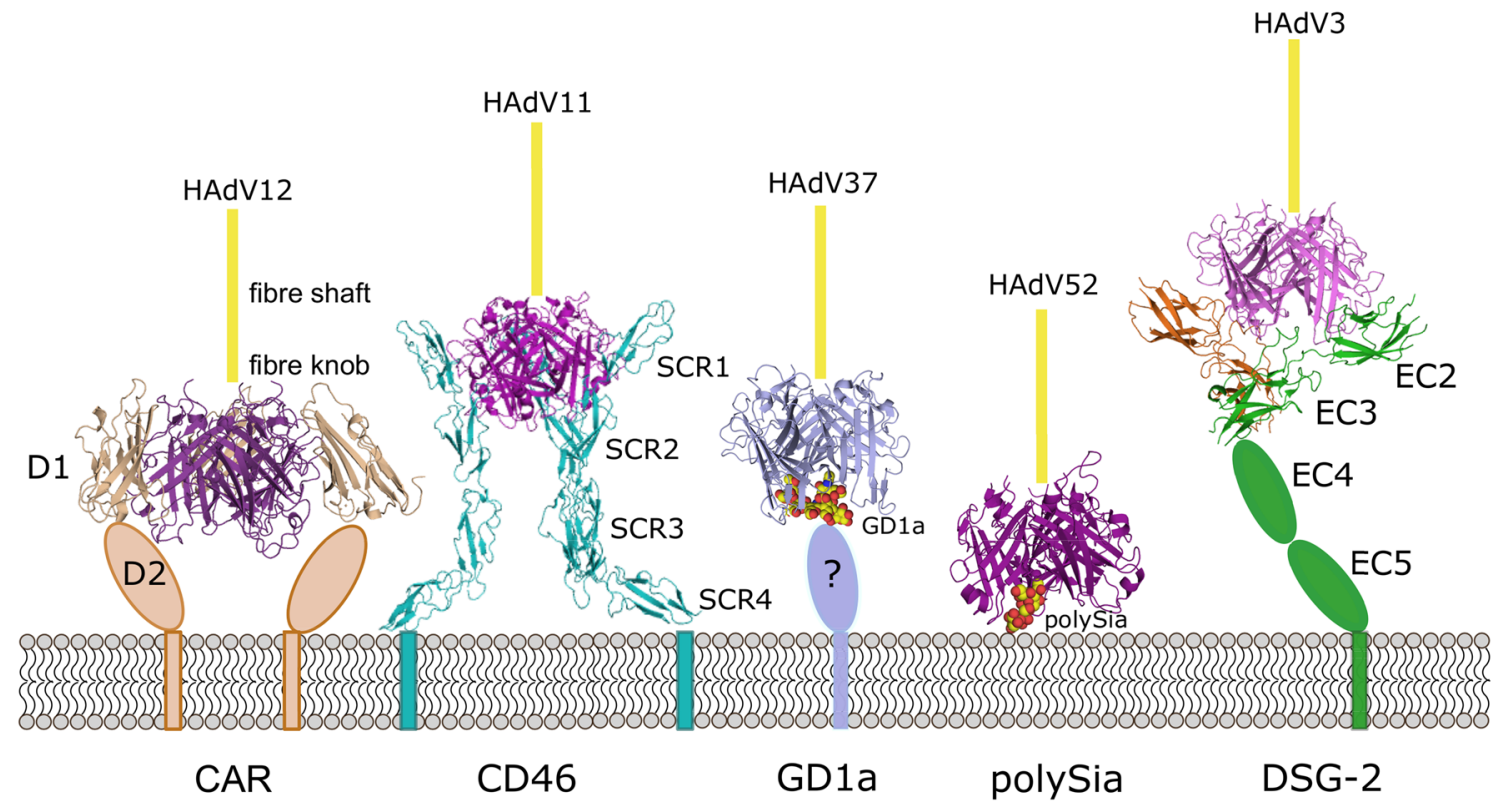

Fig. 2 Human adenovirus knob binding to attachment receptors. The trimeric knob is shown in shades of purple, the trimeric fibre shaft is represented by a yellow line (length not to scale). Domains not featured in structures are represented with cartoon shapes. CAR-D1 is bound to HAdV-12, PDB ID: 1KAC [8], CD46 to HAdV-11, PDB ID: 3O8E [39], GD1a to HAdV-37, PDB ID: 3NOI, [11], polySia to HAdV-52, PDB ID: 6G47 and two copies of DSG-2 to HAdV-3, PDB ID: $6 \mathrm{QNU}$ 
observed between the two domains. However, when interacting with the HAdV-11 knob, SCR 1 becomes almost colinear with SCR2 [25]. The interface of CD46 and this knob is remarkably long and flat, with the binding mediated not only by loops but also main-chain interactions and $\pi-\pi$ stacking of crucial arginine residues, including a conserved Arg280 in the knob. HAdV-11 binds to CD46 with high affinity and comparisons between the structure of its fibre knob and that of the lower affinity group B HAdVs (HAdV 7, 14, 21) have provided insight into mechanisms and determinants of the interaction with CD46. These include absence of an Arg residue next to $\operatorname{Arg} 280$ involved in the stacking [40] and a change in position of surface loops which disrupt the receptor-virus interface [41]. In all fibre knob-CD46 complexes, the binding is symmetrical and involves one CD46 per one knob monomer. All CD46 molecules make identical contacts with the three fiber knob monomers (see Fig. 2).

\section{Glycans: GD1a and polysialic acid}

Glycans, or carbohydrates, constitute an essential class of molecules that is known to serve a wide range of physiological functions [42]. Glycans can be conjugated to other, non-saccharide moieties, such as proteins or lipids, and their diversity in structure, as well as chemical modifications, enables them to mediate functions in signalling, adhesion, and developmental processes. Their prominent display on many cell surfaces makes them a target for numerous pathogens [42].

The first structural data showing interactions of HAdVs with glycans identified three sialic-acid binding sites at the top of the fibre knobs of both HAdV-37 and HAdV-19p (prototypical), around the central cavity [43]. Sialic acid is a monosaccharide derived from neuraminic acid, which serves a range of functions, for instance in immune regulation, and is commonly found on cell surfaces [44]. Sialic acid-containing molecules are employed as receptors by numerous viruses, including influenza viruses, rotaviruses, polyomaviruses, paramyxoviruses, and HAdVs, primarily from group D [45]. In HAdVs, the sialic acid residues bound were moieties of a sialyl-lactose ligand, and both $\alpha(2,3)$ - and $\alpha(2,6)$-linked ones could bind the knob. Unlike for CD46 and CAR, the binding sites for sialic acid were located on top of the fibre knob, one per knob monomer [43]. However, identification of the full glycan ligand of HAdV-37 as the GD1 a glycan showed that two binding sites could be occupied by a single receptor [11] (see Fig. 2).

GD1a is a ganglioside (a glycosphingolipid) prominent in the mammalian nervous system [46]. The GD1a ganglioside is not the HAdV receptor itself, but rather the GDla glycan is thought to be attached to a glycoprotein with an up to now unidentified protein conjugate. GD1a is Y-shaped and contains two terminal sialic acids, which engage the fibre knob at the top in a very similar manner for both. The sialic acid carboxylate group forms hydrogen bonds with residue Lys345, which was experimentally shown to be crucial for sialic-acid binding [11]. Residues from two fibre knob monomers are involved in binding one sialic acid moiety: one monomer provides hydrogen bonds and the other contributes van der Waals interactions and water-mediated hydrogen bonds, as well as water-mediated hydrogen bonds to galactose moieties anterior to the sialic acids. These glycan moieties bond to sialic acid pointed upwards, away from the binding site, thus leaving space for longer chains terminating in a sialic acid [11]. Knobs that bind sialic-acid are characterised by a highly positive electrostatic potential, which is thought to complement the negative charges of the receptor. No conformational change is seen on binding [43].

The divalent GD1a receptor engages two out of three possible binding sites in the knob. This asymmetry of binding differentiates the GD1a receptor from CAR and CD46, although it is not a unique feature among the HAdV receptors (see below).

Another HAdV type which uses a glycan as its receptor is HAdV-52, a member of group $\mathrm{G}$, which has two different types of fibres: a short fibre and a long fibre, that are present in a 1:1 ratio on the virion [18]. The knob of the long fibre binds to CAR, while the knob of the short fibre binds polysialic acid, and this latter compound is the major attachment receptor for this virus type. The first crystal structure of short fibre knob in complex with a glycan bound 2-O-methyl-sialic acid and showed that the binding site is distinct from the one seen in HAdV-37. It is located where the EG and GH loops from two monomers come into contact, and there are again three identical binding sites per knob. The interactions between the knob and the receptor are hydrogen bond-mediated both by the backbone and side chains of the short fibre knob. The conserved RGN motif on the GH loop is of importance for this, although it seems exclusive to HAdV-52 [47]. The receptor was identified as $\alpha(2,8)$-linked polysialic acid, and the structure obtained by X-ray crystallography showed the importance of transient electrostatic interactions with long glycan chains for the binding [18].

Polysialic acid (polySia) has been linked to many developmental functions, particularly in the human nervous system [48]. While only the non-reducing end was shown to interact with the knob in a stable manner, less stable and less directed electrostatic interactions were shown to also be of consequence to its binding [18]. A positively-charged border around the binding site and the binding site itself mediates contact with polyanionic sialic acid residues further away from the non-reducing end. Due to the transient nature of these interactions, they were not clearly visible in the structure, but simulations suggested that beyond a fifth sialic acid residue these interactions no longer increase. The 
ability to bind sialic acid chains of different lengths may also assist in infecting different cell types and hosts. An arginine residue was also shown to be crucial to polySia binding by maintaining the appropriate charge, and three polySia binding was symmetrical.

In addition to the two binding sites described above, it is also worth noting that a third sialic acid binding site has been identified in canine adenovirus 2 [49], again highlighting the diversity and adaptability of the adenovirus fibre knob to variable receptors.

\section{Desmoglein-2}

DSG-2 is a transmembrane glycoprotein that belongs to the cadherin family and is involved in maintaining cell-cell adhesion in structures termed desmosomes, which are particularly important in tissues undergoing significant mechanical stress (e.g. heart muscle) [50]. In these junctions, adhesion is mediated by DSG-2 forming heterodimers with a related protein, desmocollin [51]. DSG-2 overexpression has been observed in a number of cancers, and the protein has been identified as a receptor for some group B HAdVs, that have been classified as group B-2 and include HAdV 3, 7, 11 and 14 [9].

The structure of the extracellular fragment of DSG-2 shows that the protein comprises five extracellular cadherin domains (EC1-5) in addition to a transmembrane segment and an intracellular domain [51]. These five domains (with EC1 distal to the cell membrane, and EC5 the most proximal) are linked linearly by $\mathrm{Ca} 2+$ ion-binding regions, and they form a curved shape due to a $100^{\circ}$ bend between EC3 and EC4. One side of the protein is extensively glycosylated. In the crystal structure, DSG-2 forms homodimers (unlike the desmocollin-containing heterodimers favoured in vivo), linked by strand-swapping mechanisms [51].

A recently published cryo-electron microscopy (cryoEM) structure of the fibre knob-binding domains (EC2 and EC3) of DSG-2 in complex with the HAdV-3 fibre knob reveals that this interaction is also an example of a non-symmetrical receptor-fibre knob interaction [52]. In fact, two distinct stoichiometries of binding are observed: a 1:1, and a 2:1 receptor to fibre knob ratio (see Fig. 2). Importantly, no 3:1 binding is seen, indicating that the trimeric knob cannot engage three DSG-2 molecules at the same time, perhaps because of steric hindrance.

DSG-2 binds at the centre-top of the fibre knob, and in the 1:1 complex it interacts with two out of three monomers. The interactions are primarily mediated by knob loops, with one DSG-2 monomer contacting one knob monomer. The EC2 domain has a stabilising function, while EC3 participates in most of the interactions. Like for CD46, a positional shift is observed in the DSG-2 fragment on fibre knob binding: $\mathrm{EC} 2$ rotates by about $10^{\circ}$, once the first, essential interaction with EC3 has been established.

In the 2:1 complex the binding of the second DSG-2 molecule is mediated by very similar contacts, although the limited resolution ( 3.5 and $3.8 \AA$ ) makes it challenging to identify them and their nature clearly. An Asp261 fibre knob residue which is presumed to stabilise loop conformation was shown to be essential to receptor binding. However, it is doubtful that such 2:1 binding mode would be possible in vivo, among the desmocollin-DSG-2 heterodimers in cell-cell junctions [52].

The structural data on DSG-2 binding to HAdV show that the knob is likely too small, and the binding sites for DSG-2 are too close to each other, to allow for simultaneous binding of three DSG-2 molecules to the same knob. The observed asymmetrical, stoichiometrically non-uniform binding of DSG-2 to the fibre knob is of particular interest to studies of integrin-penton base binding (see below), which is also prone to forming asymmetric complexes of different stoichiometries. This decreases the chances of successfully obtaining crystals for X-ray studies, and increases the challenges of cryo-EM data processing as different forms of the complex need to be processed separately.

\section{HAdV interactions with entry receptors- proteins of the integrin family}

Integrins are a family of heterodimeric transmembrane proteins involved in signalling between the cell and its environment. They influence processes such as growth, development, immunity, and have been implicated in cancer [53]. Integrins consist of two extracellular subunits, the $\alpha$ and $\beta$ chains, which each have single transmembrane domains and short cytoplasmic tails [54]. Eighteen different $\alpha$ and eight different $\beta$ subunits have been identified in vertebrates, combining into 24 proteins. These subunits are composed of a number of smaller domains, which enable a remarkable degree of conformational flexibility of the protein: from a bent to an extended open conformation (see Fig. 3a). This shift is associated with activation of signalling [53].

Due to their ubiquity, integrins are exploited by a number of viruses as receptors, including the foot-and-mouth disease virus, members of the herpes virus family, reoviruses and the human papillomavirus-16 [55]. Adenoviruses also employ integrins as entry receptors. They engage integrins via the penton protein, and a penton-integrin interaction following attachment seems to be uniform among HAdVs. Interactions with integrins stimulate integrin clustering signalling for endocytosis, and virus entry into the host cell via the endocytic pathway is thought to be promoted through such receptor clustering. The integrin most extensively studied in the context of HAdV entry is $\alpha_{v} \beta_{3}$ [22], although other 


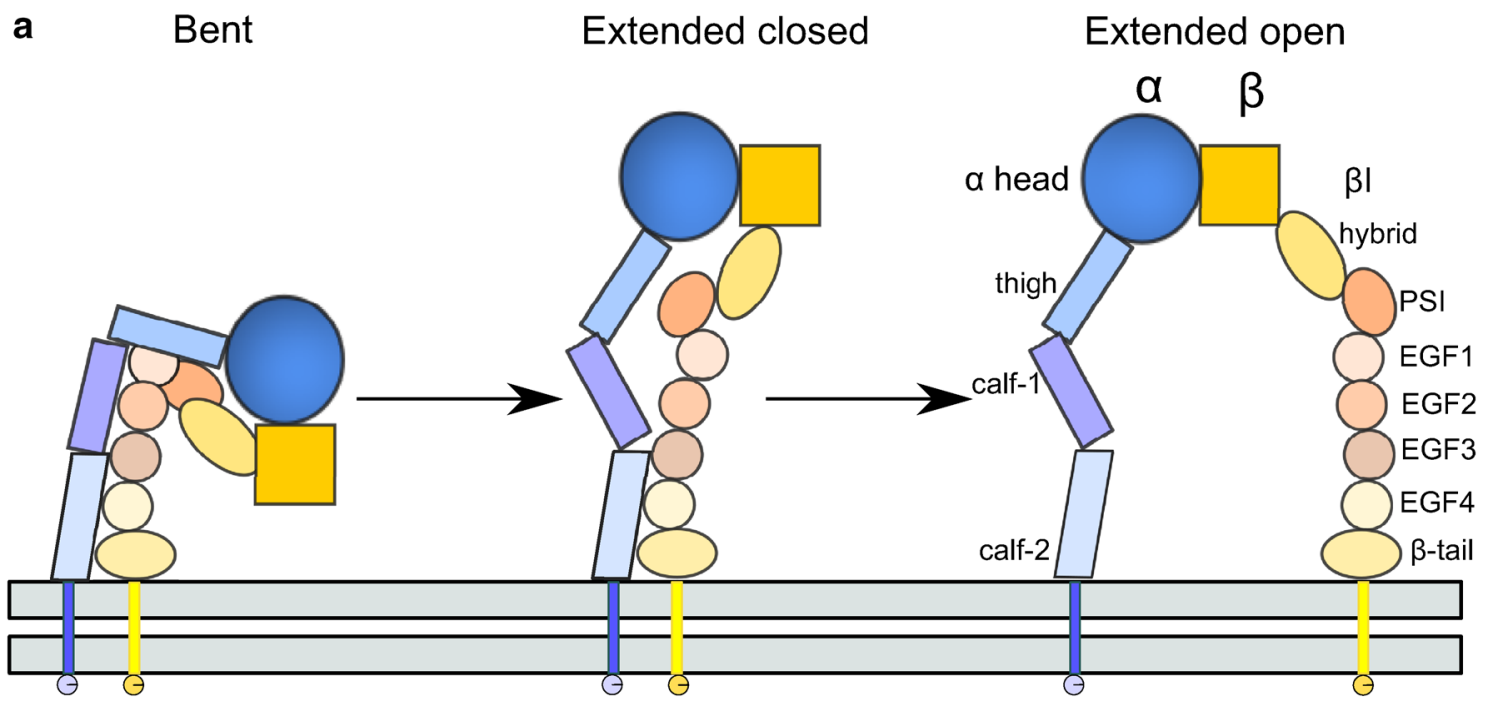

b

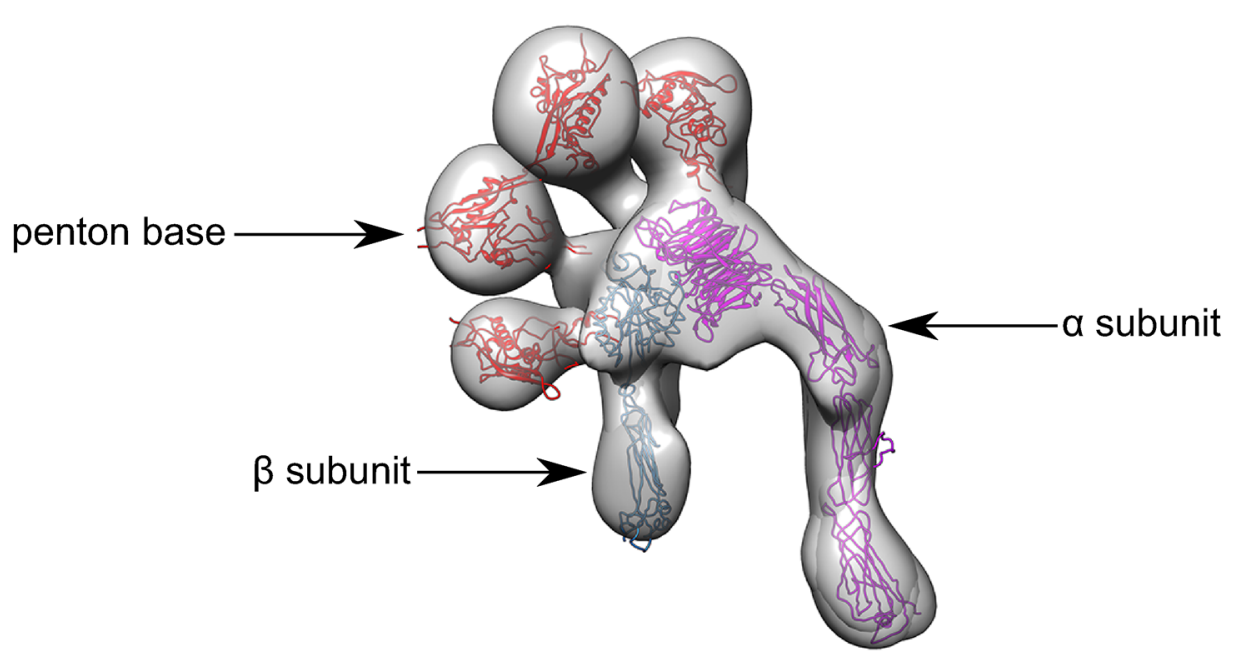

Fig. 3 a A cartoon representation of integrin conformational changes. b The different binding modes for HAdV-9 monomeric penton base to integrin $\alpha_{v} \beta_{3}$. The penton base is in red, the integrin $\alpha$ subunit is in purple, and the $\beta$ subunit in blue. EMDB IDs: 5955-5973 [58]

integrins, such as $\alpha_{v} \beta_{5}, \alpha_{v} \beta_{1}$ and $\alpha_{3} \beta_{1}[22,56]$ can also function in the same manner.

The first crystal structure of the integrin $\alpha_{\mathrm{v}} \beta_{3}$ ectodomain showed a bent conformation, with a globular "head" where the N-terminals of the two subunits meet, then separating into two more loosely associated "legs" [57]. The $\alpha_{v}$ head consists of a seven-bladed $\beta$-propeller fold, while a metal ion-dependent adhesion site (MIDAS) of the $\beta$ subunit mediates the binding between two legs at the "knee", contributing to the conformational change between bent and open conformation via shifts in the position of loops. The RGD motif-binding site is located in the head of the $\beta$ subunit.

Integrins bind to the RGD motif on the flexible HAdV penton base loop (with the exception of HAdV-40 and 41, which lack the RGD motif and interact with integrins via an unknown mechanism [24]). While the pentameric penton base consists of five pentons and hence contains five RGDcontaining loops, it is highly improbable that five integrins could bind the penton simultaneously due to steric clashes [58]. However, given the importance of integrin clustering for HAdV entry signalling, binding of multiple integrins is expected, probably with variable stoichiometries in solution. Given this asymmetry and heterogeneity, it is unlikely that high-quality crystals of a single type of HadV-integrin complex can be obtained, and electron microscopy is, therefore, the method of choice, as one can distinguish different complexes and process them separately. To date, two studies of whole virus complexes with different integrin receptors $[59,60]$, and one of monomeric penton base in complex with the integrin [58] have been published.

The imposition of icosahedral symmetry and lack of highresolution structures of the integrin and penton base to dock 
into the density at that time meant that it was initially reasoned that a full occupancy of five integrins would be present around the penton. This had been based on structures of HAdV-12 (group A) and HAdV-2 (group C) in complex with integrin $\alpha_{v} \beta_{5}$ at a resolution of about $20 \AA$, based on a ring of density above the pentamer and SPR measurements showing binding of 4.2 integrins per HAdV-2 pentamer [59]. However, after high-resolution integrin and penton base structures had been published it was recognised that at most four integrins, seen in an extended conformation and in different orientations, could fit around the HAdV-12 penton without steric clashes. The more flexible and extendable RGD loop of HAdV-2 penton was speculated to potentially accommodate five integrins [60].

It was also suggested that the fourth integrin would require a conformational shift in the penton base against the inter-domain "twist", and that this change may be responsible for the penton's disassociation from the capsid during HAdV entry and release of the fibre knob [60]. The different integrin orientations and varying occupancies contribute to asymmetry, making the complex structure more challenging to solve with the imposition of symmetry.

Single-particle reconstruction of negative-stain EM monomeric HAdV-9 (group D) penton base insertion domain only integrin $\alpha_{v} \beta_{3}$ showed that a variety of integrin conformations could bind the penton, from bent to extended [58]. Moreover, the monomeric penton was shown to bind the integrin in a number of different locations at the integrin head, showing the interactions to be more varied than expected (see Fig. 3b). It is unclear as yet if this variability is present in the more sterically constrained conditions of virus binding to host cell, and if the integrin conformational flexibility is restrained by the presence of transmembrane and intracellular domains.

\section{Outlook}

HAdVs are remarkable in the diversity of attachment receptors they employ, which is a contributing factor to their broad cellular tropism. Studies of their receptor-virus interactions using structural biology techniques have significantly advanced our understanding of HAdV attachment and entry. This knowledge can be used both in drug design, to combat the virus as a pathogen, and in rational gene vector development, to enhance the virus as a therapeutic agent. Prospects for the field include addressing the imbalance in data on particular receptors: while some of the attachment receptors have been extensively studied in their viral context (e.g. CAR, CD46), there is much less information available on others. Moreover, the integrin entry receptors, representing the second step of HAdV infection, remain to be examined more closely, as we lack a detailed view of the contacts that are being formed in this complex, and we also lack an understanding of how the geometry of binding dictates the stoichiometry of the interaction.

Acknowledgements ACS is funded by Viral and Bacterial Adhesin Network Training Innovative Training Network, part of the European Union's Horizon 2020 Research and Innovation Programme under the Marie Skłodowska-Curie Grant Agreement No. 765042. TS acknowledges support from the Baden-Württemberg Foundation (Glycobiology/Glycomics program).

Open Access This article is distributed under the terms of the Creative Commons Attribution 4.0 International License (http://creativeco mmons.org/licenses/by/4.0/), which permits unrestricted use, distribution, and reproduction in any medium, provided you give appropriate credit to the original author(s) and the source, provide a link to the Creative Commons license, and indicate if changes were made.

\section{References}

1. Ghebremedhin B (2014) Human adenovirus: viral pathogen with increasing importance. Eur J Microbiol Immunol 4:26-33. https ://doi.org/10.1556/EuJMI.4.2014.1.2

2. Flint SJ, Enquist LW, Racaniello VR, Skalka AM (2004) Adenoviruses. Principles of virology, 2nd edn. ASM Press, Washington, DC, pp 804-805, 846

3. Kremer EJ, Nemerow GR (2015) Adenovirus tales: from the cell surface to the nuclear pore complex. PLoS Pathog 11:1-8. https ://doi.org/10.1371/journal.ppat.1004821

4. Arnberg N (2012) Adenovirus receptors: implications for targeting of viral vectors. Trends Pharmacol Sci 33:442-448. https://doi. org/10.1016/j.tips.2012.04.005

5. Baker A, Aguirre-Hernández C, Halldén G, Parker A (2018) Designer oncolytic adenovirus: coming of Age. Cancers (Basel) 10:201. https://doi.org/10.3390/cancers10060201

6. Dong W, Van Ginkel JWH, Au KY et al (2014) ORCA-010, a novel potency-enhanced oncolytic adenovirus, exerts strong antitumor activity in preclinical models. Hum Gene Ther 25:897-904. https://doi.org/10.1089/hum.2013.229

7. Nemerow GR, Stewart PL, Reddy VS (2012) Structure of human adenovirus. Curr Opin Virol 2:115-121. https://doi.org/10.1016/j. coviro.2011.12.008

8. Bewley MC (1999) Structural analysis of the mechanism of adenovirus binding to its human cellular receptor, CAR. Science 286:1579-1583. https://doi.org/10.1126/science.286.5444.1579

9. Wang H, Li ZY, Liu Y et al (2011) Desmoglein 2 is a receptor for adenovirus serotypes 3, 7, 11 and 14. Nat Med 17:96-104. https ://doi.org/10.1038/nm.2270

10. Marttila M, Persson D, Gustafsson D et al (2005) CD46 is a cellular receptor for all species b adenoviruses except types 3 and 7 . J Virol 79:14429-14436. https://doi.org/10.1128/jvi.79.22.14429 $-14436.2005$

11. Nilsson EC, Storm RJ, Bauer J et al (2011) The GD1a glycan is a cellular receptor for adenoviruses causing epidemic keratoconjunctivitis. Nat Med 17:105-109. https://doi.org/10.1038/nm.2267

12. Henry LJ, Xia D, Wilke ME et al (1994) Characterization of the knob domain of the adenovirus type 5 fiber protein expressed in Escherichia coli. J Virol 68:5239-5246

13. Xia D, Henry LJ, Gerard RD, Deisenhofer J (1994) Crystal structure of the receptor-binding domain of adenovirus type 5 fiber 
protein at 1.7 A resolution. Structure 2:1259-1270. https://doi. org/10.1016/s0969-2126(94)00126-x

14. van Raaij MJ, Mitraki A, Lavigne G, Cusack S (1999) A triple $\beta$-spiral in the adenovirus fibre shaft reveals a new structural motif for a fibrous protein. Nature 401:935-938. https://doi. org/10.1038/44880

15. Cao C, Dong X, Wu X et al (2012) Conserved fiber-penton base interaction revealed by nearly atomic resolution cryo-electron microscopy of the structure of adenovirus provides insight into receptor interaction. J Virol 86:12322-12329. https://doi. org/10.1128/jvi.01608-12

16. Bergelson JM, Droguett G, Kurt-jones EA et al (1997) Isolation of a common receptor for coxsackie B viruses and adenoviruses 2 and 5. Science 275:1320-1324. https://doi.org/10.1126/scien ce. 275.5304 .1320

17. Tomko RP, Xu R, Philipson L (1997) HCAR and MCAR: the human and mouse cellular receptors for subgroup $\mathrm{C}$ adenoviruses and group B coxsackieviruses. Proc Natl Acad Sci USA 94:3352-3356. https://doi.org/10.1073/pnas.94.7.3352

18. Lenman A, Liaci AM, Liu Y et al (2018) Polysialic acid is a cellular receptor for human adenovirus 52. Proc Natl Acad Sci 115:E4264-E4273. https://doi.org/10.1073/pnas.1716900115

19. Lenman A, Muller S, Nygren MI et al (2011) Coagulation Factor IX mediates serotype-specific binding of species a adenoviruses to host cells. J Virol 85:13420-13431. https://doi.org/10.1128/ jvi.06088-11

20. Jonsson MI, Lenman AE, Frangsmyr L et al (2009) Coagulation Factors IX and $\mathrm{X}$ enhance binding and infection of adenovirus types 5 and 31 in human epithelial cells. J Virol 83:3816-3825. https://doi.org/10.1128/jvi.02562-08

21. Nemerow GR, Stewart PL (2016) Insights into adenovirus uncoating from interactions with integrins and mediators of host immunity. Viruses 8:1-15. https://doi.org/10.3390/v8120 337

22. Wickham TJ, Mathias P, Cheresh DA, Nemerow GR (1993) Integrins $\alpha v \beta 3$ and $\alpha v \beta 5$ promote adenovirus internalization but not virus attachment. Cell 73:309-319. https://doi.org/10.1016/00928674(93)90231-E

23. Zubieta C, Schoehn G, Chroboczek J, Cusack S (2005) The structure of the human adenovirus 2 penton. Mol Cell 17:121-135. https://doi.org/10.1016/j.molcel.2004.11.041

24. Rajan A, Persson BD, Frängsmyr L et al (2018) Enteric species $\mathrm{f}$ human adenoviruses use laminin-binding integrins as Coreceptors for infection of Ht-29 Cells. Sci Rep 8:1-14. https://doi. org/10.1038/s41598-018-28255-7

25. Persson BD, Reiter DM, Marttila M et al (2007) Adenovirus type 11 binding alters the conformation of its receptor CD46. Nat Struct Mol Biol 14:164-166. https://doi.org/10.1038/nsmb1190

26. Cupelli K, Stehle T (2011) Viral attachment strategies: the many faces of adenoviruses. Curr Opin Virol 1:84-91. https://doi. org/10.1016/j.coviro.2011.05.024

27. Ortiz-Zapater E, Santis G, Parsons M (2017) CAR: a key regulator of adhesion and inflammation. Int J Biochem Cell Biol 89:1-5. https://doi.org/10.1016/j.biocel.2017.05.025

28. van Raaij MJ, Chouin E, van der Zandt $\mathrm{H}$ et al (2000) Dimeric structure of the coxsackievirus and adenovirus receptor D1 domain at $1.7 \AA$ resolution. Structure 8:1147-1155. https://doi. org/10.1016/S0969-2126(00)00528-1

29. Matthäus C, Langhorst H, Schütz L et al (2017) Cell-cell communication mediated by the CAR subgroup of immunoglobulin cell adhesion molecules in health and disease. Mol Cell Neurosci 81:32-40. https://doi.org/10.1016/j.mcn.2016.11.009

30. Jiang S, Jacobs A, Laue TM, Caffrey M (2004) Solution structure of the coxsackievirus and adenovirus receptor domain 2. Biochemistry 43:1847-1853. https://doi.org/10.1021/bi035490x
31. Kirby I, Davison E, Beavil AJ et al (1999) Mutations in the DG loop of adenovirus type 5 fiber knob protein abolish high-affinity binding to its cellular receptor CAR. J Virol 73:9508-9514

32. Kirby I, Davison E, Beavil AJ et al (2000) Identification of contact residues and definition of the CAR-binding site of adenovirus Type 5 fiber protein. J Virol 74:2804-2813. https://doi. org/10.1128/jvi.74.6.2804-2813.2000

33. Jakubczak JL, Rollence ML, Stewart DA et al (2001) Adenovirus Type 5 viral particles pseudotyped with mutagenized fiber proteins show diminished infectivity of coxsackie B-adenovirus receptor-bearing cells. J Virol 75:2972-2981. https://doi. org/10.1128/jvi.75.6.2972-2981.2001

34. Baker AT, Greenshields-Watson A, Coughlan L et al (2019) Diversity within the adenovirus fiber knob hypervariable loops influences primary receptor interactions. Nat Commun 10:741. https://doi.org/10.1038/s41467-019-08599-y

35. Riley-Vargas RC, Gill DB, Kemper C et al (2004) CD46: expanding beyond complement regulation. Trends Immunol 25:496-503. https://doi.org/10.1016/j.it.2004.07.004

36. Cardone J, Le Friec G, Kemper C (2011) CD46 in innate and adaptive immunity: an update. Clin Exp Immunol 164:301-311. https://doi.org/10.1111/j.1365-2249.2011.04400.x

37. Bergelson JM, Chan M, Solomon KR et al (1994) Decay-accelerating factor (CD55), a glycosylphosphatidylinositol-anchored complement regulatory protein, is a receptor for several echoviruses. Proc Natl Acad Sci 91:6245-6248. https://doi.org/10.1073/ pnas.91.13.6245

38. Fingeroth JD, Weis JJ, Tedder TF et al (1984) Epstein-Barr virus receptor of human $\mathrm{B}$ lymphocytes is the $\mathrm{C} 3 \mathrm{~d}$ receptor $\mathrm{CR} 2$. Proc Natl Acad Sci USA 81:4510-4514

39. Persson BD, Schmitz NB, Santiago C et al (2010) Structure of the extracellular portion of CD46 provides insights into its interactions with complement proteins and pathogens. PLoS Pathog 6:e1001122. https://doi.org/10.1371/journal.ppat.1001122

40. Persson BD, Muller S, Reiter DM et al (2009) An arginine switch in the species B adenovirus knob determines high-affinity engagement of cellular receptor CD46. J Virol 83:673-686. https://doi. org/10.1128/JVI.01967-08

41. Cupelli K, Muller S, Persson BD et al (2010) Structure of adenovirus type 21 knob in complex with CD46 reveals key differences in receptor contacts among species B adenoviruses. J Virol 84:3189-3200. https://doi.org/10.1128/jvi.01964-09

42. Varki A (2017) Biological roles of glycans. Glycobiology 27:349. https://doi.org/10.1093/glycob/cww086

43. Burmeister WP, Guilligay D, Cusack S et al (2004) Crystal structure of species D adenovirus fiber knobs and their sialic acid binding sites. J Virol 78:7727-7736. https://doi.org/10.1128/ jvi.78.14.7727-7736.2004

44. Varki A (2007) Glycan-based interactions involving vertebrate sialic-acid-recognizing proteins. Nature 446:1023-1029. https:// doi.org/10.1038/nature05816

45. Stencel-Baerenwald JE, Reiss K, Reiter DM et al (2014) The sweet spot: defining virus-sialic acid interactions. Nat Rev Microbiol 12:739-749. https://doi.org/10.1038/nrmicro3346

46. Schnaar RL (2016) Gangliosides of the vertebrate nervous system. J Mol Biol 428:3325-3336. https://doi.org/10.1016/j. jmb.2016.05.020

47. Lenman A, Liaci AM, Liu Y et al (2015) Human adenovirus 52 uses sialic acid-containing glycoproteins and the coxsackie and adenovirus receptor for binding to target cells. PLoS Pathog 11:1-23. https://doi.org/10.1371/journal.ppat.1004657

48. Galuska C, Lütteke T, Galuska S (2017) Is polysialylated NCAM not only a regulator during brain development but also during the formation of other organs? Biology (Basel) 6:27. https://doi. org/10.3390/biology6020027 
49. Rademacher C, Bru T, McBride R et al (2012) A Siglec-like sialic-acid-binding motif revealed in an adenovirus capsid protein. Glycobiology 22:1086-1091. https://doi.org/10.1093/glyco b/cws073

50. Dusek RL, Godsel LM, Green KJ (2007) Discriminating roles of desmosomal cadherins: beyond desmosomal adhesion. J Dermatol Sci 45:7-21. https://doi.org/10.1016/j.jdermsci.2006.10.006

51. Harrison OJ, Brasch J, Lasso G et al (2016) Structural basis of adhesive binding by desmocollins and desmogleins. Proc Natl Acad Sci 113:7160-7165. https://doi.org/10.1073/pnas.16062 72113

52. Vassal-Stermann E, Effantin G, Zubieta C et al (2019) CryoEM structure of adenovirus type 3 fibre with desmoglein 2 shows an unusual mode of receptor engagement. Nat Commun 10:1181. https://doi.org/10.1038/s41467-019-09220-y

53. Campbell ID, Humphries MJ (2011) Integrin structure, activation, and interactions. Cold Spring Harb Perspect Biol 3:a004994. https ://doi.org/10.1101/cshperspect.a004994

54. Luo B-H, Carman CV, Springer TA (2007) Structural basis of integrin regulation and signaling. Annu Rev Immunol 25:619647. https://doi.org/10.1146/annurev.immunol.25.022106.141618

55. Hussein HAM, Walker LR, Abdel-Raouf UM et al (2015) Beyond RGD: virus interactions with integrins. Arch Virol 160:26692681. https://doi.org/10.1007/s00705-015-2579-8
56. Storm RJ, Persson BD, Skalman LN et al (2017) Human Adenovirus Type 37 Uses $\alpha \mathrm{V} \beta 1$ and $\alpha 3 \beta 1$ integrins for infection of human corneal cells. J Virol 91:2019-2020. https://doi.org/10.1128/ JVI.02019-16

57. Xiong J, Stehle T, Diefenbach B et al (2001) Crystal structure of the extracellular segment of integrin $\alpha \mathrm{V} \beta 3$. Science 294:339-345. https://doi.org/10.1126/science.1064535.Crystal

58. Veesler D, Cupelli K, Burger M et al (2014) Single-particle EM reveals plasticity of interactions between the adenovirus penton base and integrin $\alpha V \beta 3$. Proc Natl Acad Sci 111:8815-8819. https ://doi.org/10.1073/pnas.1404575111

59. Chiu CY, Mathias P, Nemerow GR, Stewart PL (1999) Structure of adenovirus complexed with its internalization receptor, $\alpha v \beta 5$ integrin. J Virol 73:6759-6768

60. Lindert S, Silvestry M, Mullen TM et al (2009) Cryo-electron microscopy structure of an adenovirus-integrin complex indicates conformational changes in both penton base and integrin. J Virol 83:11491-11501. https://doi.org/10.1128/jvi.01214-09

Publisher's Note Springer Nature remains neutral with regard to jurisdictional claims in published maps and institutional affiliations. 